quite true that trauma to the lumbosacral spine can result in bilateral foot drop, but this was ruled out in the preanesthetia visit and the magnetic resonance imaging done postoperatively. The patient did not have any other coexisting disease with subclinical neuropathy (diabetes), although pre-existing neurological disease should also be ruled out e.g., lumbar disc syndrome or spondylitis or recent herpes zoster in such cases. The tibial pins were inserted on both sides, and the femur alone was operated during the first stage. The legs were surgically reduced in the second stage, so the foot drop could easily be related to the surgery. The patient was operated in the lateral position, no femoral traction was applied (on the hip table). We agree that the sciatic nerve compression in the lateral position could again be a factor (but unilateral).

Since the patient had no neurological deficit preoperatively, a neurologist's opinion was not sought. Consultation was undertaken postoperatively, after the patient developed foot drop. There was no documentation of the presence of foot drop at admission or after the insertion of tibial pins (bilateral) so the etiology was most likely related to surgery or spinal anesthesia. Bilateral foot drop after spinal anesthesia is possible only if lumbar puncture is performed above the third lumbar segment, which is uncommon. Considering the angle of entry of the spinal needle, trauma to one side of the conus is possible.

We emphasize that this case is not meant to attribute blame, but to highlight that this complication, though rare, does occur, and has its own medicolegal implications.

Anju Ghai MD

Sarla Hooda MD

Prashant Kumar MD

Raman Kumar MD

Pankaj Bansal MD

Sharma Post Graduate Institute of Medical Sciences, Haryana, India

E-mail: prashantkumarz@yahoo.com

\section{Does bilateral thoracic sympathectomy predispose to reflex bronchospasm fol- lowing tracheal intubation?}

To the Editor:

Thoracic endoscopic sympathectomy has become the technique of choice for treating intractable essential hyperhidrosis. ${ }^{1}$ We report severe bronchospasm following tracheal intubation in a patient with a previous history of bilateral thoracic sympathectomy. Consent for publication was obtained from the patient.
A 31-yr-old non-smoking woman, $60 \mathrm{~kg}$, without a history of allergy, or asthma, was scheduled for left knee arthroscopy. Two months previously, she had an uneventful general anesthetic for bilateral thoracoscopic sympathectomy to treat essential hyperhidrosis. Preoperative examination revealed clear lungs. Following preoxygenation, anesthesia was induced with propofol $2.5 \mathrm{mg} \cdot \mathrm{kg}^{-1} i v$, vecuronuim $0.15 \mathrm{mg} \mathrm{kg}^{-1} i v$, and fentanyl $2 \mu \mathrm{g} \cdot \mathrm{kg}^{-1} i v$. Ventilation by facemask was easy. Following onset of neuromuscular block, tracheal intubation was easily performed by direct laryngoscopy. Immediately following intubation, ventilation became difficult. Chest auscultation revealed bilateral expiratory wheezing associated with decreased air entry and increased airway pressure up to $60 \mathrm{~cm} \mathrm{H}_{2} 0$. Oxygen saturation, as monitored by pulse oximetry, decreased from $100 \%$ to $80 \%$. Anesthesia was deepened by inhalation of sevoflurane (4-8\% inspired concentration) in $100 \%$ oxygen. Six doses of $20 \mu \mathrm{g}$ ipratropium bromure and five doses of $100 \mu \mathrm{g}$ salbutamol were administered via the endotracheal tube. During the following five minutes, ventilation became progressively easier, oxygen saturation increased to $100 \%$, and breath sounds normalized. Anesthesia was continued with $2 \%$ to $4 \%$ sevoflurane. Throughout this event, no cutaneous flushing was observed, blood pressure ranged between $110 / 90 \mathrm{mmHg}$ and $130 / 95 \mathrm{mmHg}$, with pulse rate between 65 and 115 beats. $\mathrm{min}^{-1}$. Surgery and recovery proceeded uneventfully. Twelve weeks after surgery, an atopy skin patch test was negative.

The severe bronchospasm experienced by the patient may have been secondary to an anaphylactic reaction to the induction agents. In a recent French survey of anaphylaxis, ${ }^{2}$ the most common features were cardiovascular manifestations $(71.5 \%)$, followed by cutaneous symptoms (69\%). The least frequent feature was bronchospasm which only occurred in $44.2 \%$ of the cases, and was associated with a history of atopy or asthma. In our patient, there was no history of atopy or reactive airway disease and the bronchospasm was not associated with any cardiovascular or cutaneous manifestations. The severe bronchospasm occurred immediately following tracheal intubation, suggesting that it may have been a reflex response which was triggered by instrumentation of the airway under a light level of anesthesia. Other causes of bronchospasm such as unrecognized aspiration, carinal irritation, secretions, or chemical irritants that may have contaminated the tracheal tube were also considered and excluded.

Patients with essential hyperhidrosis have sympathetic overactivity, associated with compensatory high parasympathetic tone. Sympathectomy results in 
a decrease of plasma norepinephrine, ${ }^{3}$ and parasympathetic predominance ${ }^{4}$ which may increase airway resistance..$^{5}$ Intraoperative bronchospasm is usually cholinergically-mediated. Thus, patients with essential hyperhidrosis who have undergone bilateral thoracic sympathectomy, may be more liable to develop reflex bronchospasm under light levels of anesthesia.

Ahed Zeidan $\mathrm{MD}^{*}$

Nazih Nahle MD*

Anis Baraka MD FRCA†

Sahel General Hospital, * American University of Beirut Medical Center, $†$ Beirut, Lebanon

E-mail: abaraka@aub.edu.lb

\section{References}

1 Hashmonai M, Kopelman D, Assalia A. The treatment of primary palmar hyperhidrosis: a review. Surg Today 2000; 30: 211-8.

2 Laxenaire MC, Mertes PM; Groupe d'Études des Réactions Anaphylactoides Peranesthésiques. Anaphylaxis during anaesthesia. Results of a two-year survey in France. Br J Anaesth 2001; 87: 549-58.

3 Noppen M, Sevens C, Gerlo E, Vincken W. Plasma catecholamine concentrations in essential hyperhidrosis and effects of thoracoscopic D2-D3 sympathicolysis. Eur J Clin Invest 1997; 27: 202-5.

4 Wiklund U, Koskinen LO, Niklasson U, Bjerle P, Elfversson J. Endoscopic transthoracic sympathicotomy affects the autonomic modulation of heart rate in patients with palmar hyperhidrosis. Acta Neurochir (Wien) 2000; 142: 691-6.

5 Tseng MY, Tseng JH. Thoracoscopic sympathectomy for palmar hyperhidrosis: effects on pulmonary function. J Clin Neurosci 2001; 8: 539-41.

\section{Retained and cut stimulating infra- clavicular catheter}

To the Editor:

We report the case of a stimulating infraclavicular catheter that was retained and subsequently cut, requiring a surgical incision for removal. Consent for publication of personal information was obtained from the patient according to our local institutional guidelines.

A 52-yr-old American Society Anesthesiologists class 1 woman had an open reduction and internal fixation of her left radius under general anesthesia. Postoperatively she was given narcotics for analgesia but experienced severe nausea and vomiting. She agreed to the insertion of a continuous infraclavicular brachial plexus block for pain control. Using Wilson's landmarks, ${ }^{1}$ an Arrow StimucathTM Continuous Nerve catheter (Arrow International, Reading, PA, USA) was placed uneventfully in the left infracoracoid area. The catheter was advanced easily under stimulation $4 \mathrm{~cm}$ beyond the tip of the needle. Its proximal end was tunnelled under the skin leaving a small skin bridge at the initial insertion site of the needle. The patient received a bolus of $30 \mathrm{~mL}$ bupivacaine $0.25 \%$ followed by a continuous infusion $\left(10 \mathrm{~mL} \cdot \mathrm{hr}^{-1}\right.$ ropivacaine $0.2 \%$ ) with excellent pain relief.

The following day, the local anesthetic infusion was stopped to allow a neurological assessment of the operated limb by the surgeon. As her pain was well controlled with acetaminophen and codeine, the patient requested that her catheter be removed. After experiencing resistance upon removal of the catheter, the patient's nurse cut the catheter at the skin bridge. When she pulled on the distal end of the catheter, the sheath came off, leaving the stimulating wire in place. We were then notified. After assessment, the patient was taken to the operating room. With fluoroscopy, the wire was visualized and knotting was ruled out. Under local anesthesia, a small incision was made and the wire removed (Figure).

Inability to remove perineural catheters due to knotting or breakage has been described after femoral, ${ }^{2}$ fascia iliaca, ${ }^{3}$ sciatic, ${ }^{4}$ and axillary ${ }^{5}$ blockade. Most of these cases required surgical extraction for catheter removal. We are the first to describe a retained stimulating infraclavicular catheter. The latter became separated from its stimulating wire after being cut. In this institution nurses on the ward remove all nerve catheters. We feel that it is important to instruct the nursing staff and ambulatory patients never to cut stimulating perineu-

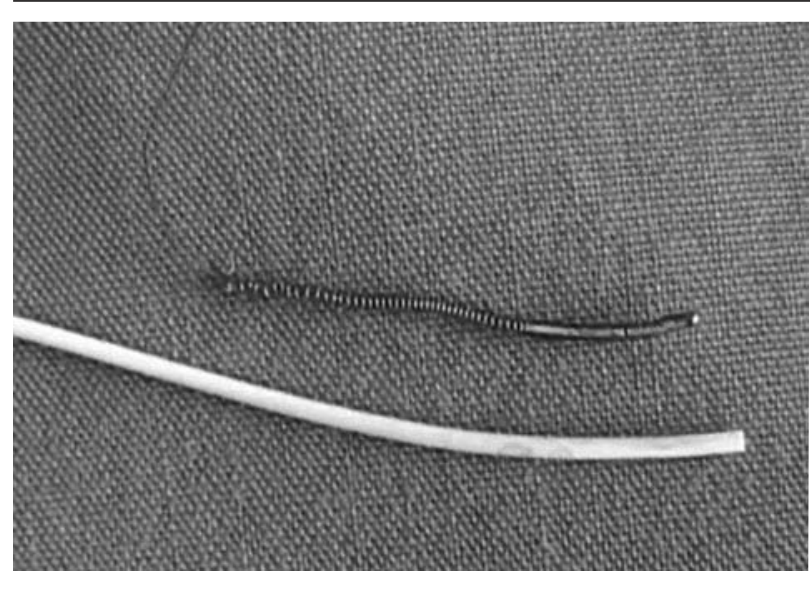

FIGURE Distal end of catheter sheath and wire. 